\title{
Violencia familiar durante el embarazo y riesgo de bajo peso en el recién nacido Hospital "El Carmen"
}

\section{Family violence during pregnancy and risk of low birth weight in newborns Hospital "El Carmen"}

\author{
Meza Victoria, Hugo ' / Quiliano Navarro, Mónica² \\ 'hugomezav@yahoo.com \\ 2monicarqn1@hotmail.com
}

Facultad de Enfermería de la Universidad Nacional del Centro del Perú

\section{RESUMEN}

El estudio titulado violencia familiar durante el embarazo y riesgo de bajo peso en el recién nacido Hospital "El Carmen", tuvo como objetivo Determinar la relación existente entre la violencia familiar durante el embarazo y el riesgo de bajo peso en el recién nacido Hospital "El Carmen" Huancayo 2014.

El estudio es de nivel aplicativo, tipo cuantitativo, método descriptivo de corte transversal, la población de estudio estuvo conformada por 40 gestantes; para la recolección de datos se utilizó la encuesta, así como la revisión de las historias clínicas. El estudio evidencia que la violencia familiar es uno de los factores principales para la presencia de recién nacidos con bajo peso al nacer para su edad gestacional; de igual forma, el grado de instrucción, el estado civil influyeron significativamente en la presencia de violencia durante el periodo de gestación. El hecho de que las mujeres no sufran de violencia familiar, a pesar de que conocen sus derechos y que por diferentes razones no los hacen respetar, pone en evidencia la deficiente promoción acerca del tema y el empoderamiento que debe tener la mujer embarazada para poder prevenir incremento de morbimortalidad materna y perinatal ; a la vez destaca la importancia de implementar nuevas e innovadoras estrategias, con

\section{ABSTRACT}

The study entitled Family Violence during pregnancy and the risk of low birth weight newborns Hospital "El Carmen", aimed to determine the association between domestic violence during pregnancy and the risk of low birth weight in newborns Hospital Carmen Huancayo 2014. The study is level application, quantitative, descriptive method of cross section. The study population consisted of 40 pregnant women; data collection for a survey designed for this, as well as the review of clinical records was used. the study finds that family violence is one of the main factors for the presence of infants with low birth weight for gestational age; similarly, educational attainment, marital status significantly influenced the presence of violence during the gestation period. The fact that women suffer domestic violence, although they know their rights and who for various reasons do not make them respect, highlights the poor promotion and education in the subject and the empowerment that pregnant women should have to prevent increased maternal and perinatal morbidity and mortality; while highlighting the importance of implementing new and innovative strategies, involving all public and private sectors, aimed at an adequate guidance on the subject responsible 
participación de todos los sectores públicos y privados, encaminadas a una adecuada orientación responsable acerca del tema violencia familiar durante el embarazo. domestic violence during pregnancy. Keywords: family violence, pregnancy.

Palabras clave: violencia familiar, embarazo.

\section{NTRODUCCIÓN}

La violencia hacia las mujeres es un problema poco reconocido por los diferentes sectores vinculados con la salud, este problema no es lo suficientemente valorado como una prioridad de la salud pública, a pesar de que es una causa importante y frecuente de morbilidad y mortalidad femeninas. La violencia hacia las mujeres embarazadas repercute en los recién nacidos, pues conduce al bajo peso al nacer (BPN) y afecta al niño en la etapa inicial de la vida, tanto por el incremento de la morbilidad y de la mortalidad como por sus efectos sobre el desarrollo de las capacidades físicas, cognoscitivas y sociales, que pueden limitar considerablemente a los que sobreviven estos episodios. Este tipo de violencia se caracteriza por un patrón de conducta coercitiva hacia las mujeres que abarca el abuso físico (golpes, quemaduras, mordeduras, heridas con arma blanca o de fuego), el abuso psicológico y emocional (intimidación, humillaciones verbales, manipulación, omisión, abandono, negligencia) y el abuso sexual, el cual consiste en forzar física o psicológicamente a la mujer a la relación sexual o a una conducta sexual de determinado tipo. Hasta la fecha, pocas investigaciones realizadas en Perú han examinado el efecto de factores psicosociales durante el embarazo en el peso de los recién nacidos. Los principales hallazgos han sido la asociación entre el bajo peso al nacer y la violencia, así como el deseo del embarazo, la sensación de seguridad de la embarazada, el apoyo y la compañía durante la gestación, como factores protectores.
La violencia hacia una mujer embarazada puede tener graves consecuencias para la madre y el feto. La violencia física o sexual que conlleva traumatismos abdominales puede provocar, según el tiempo de gestación, la pérdida del feto, el parto prematuro o bajo peso en el recién nacido.

\section{MATERIALES Y MÉTODOS}

Es un estudio de tipo descriptivo, con enfoque cuantitativo en el que no existe relación de causa efecto entre los fenómenos estudiados, y que permitió realizar un análisis de la relación que existe entre violencia familiar $v$ riesgo de bajo peso al nacer.Para el estudio se hizo uso de los métodos inductivo y deductivo así como el método descriptivo porque está orientado al conocimiento de la realidad tal como se presenta en una situación espacio temporal dada, es decir tal como se da en el presente. Es de tipo no experimental, transaccional (descriptivo correlacional.) La unidad de estudio estuvo conformada por las gestantes atendidas en el Hospital "El Carmen", y que voluntariamente aceptaron participar en la investigación.

Para la recolección de datos de la presente investigación se aplicó un instrumento elaborado por los investigadores, que recolecta información sobre violencia familiar, los mismos que midieron las variables de estudio, se consideró el consentimiento informado como principio ético. 


\section{RESULTADOS}

Tabla 1

Violencia familiar y peso para la edad gestacional del Recién nacido de las gestantes atendidas en el Hospital "El Carmen"

\begin{tabular}{|c|c|c|c|c|c|c|}
\hline \multirow{3}{*}{$\begin{array}{l}\text { PESO PARA EDAD } \\
\text { GESTACIONAL }\end{array}$} & \multicolumn{6}{|c|}{ VIOLENCIA FAMILIAR } \\
\hline & \multicolumn{2}{|c|}{ FISICA } & \multicolumn{2}{|c|}{ PSICOLOGICA } & \multicolumn{2}{|c|}{ SEXUAL } \\
\hline & FRECUENCIA & $\%$ & FRECUENCIA & $\%$ & FRECUENCIA & $\%$ \\
\hline BAJO & 15 & 54 & 19 & 48 & 1 & 33 \\
\hline INSUFICIENTE & 11 & 39 & 17 & 43 & 1 & 33 \\
\hline ADECUADO & 2 & 7 & 4 & 10 & 1 & 33 \\
\hline ALTO & 0 & 0 & 0 & 0 & 0 & 0 \\
\hline TOTAL & 28 & 100 & 40 & 100 & 3 & 100 \\
\hline
\end{tabular}

Fuente: Encuesta de la Investigación

Figura 1. Violencia familiar y peso para la edad gestacional del Recién nacido de las gestantes atendidas en el Hospital "El Carmen"

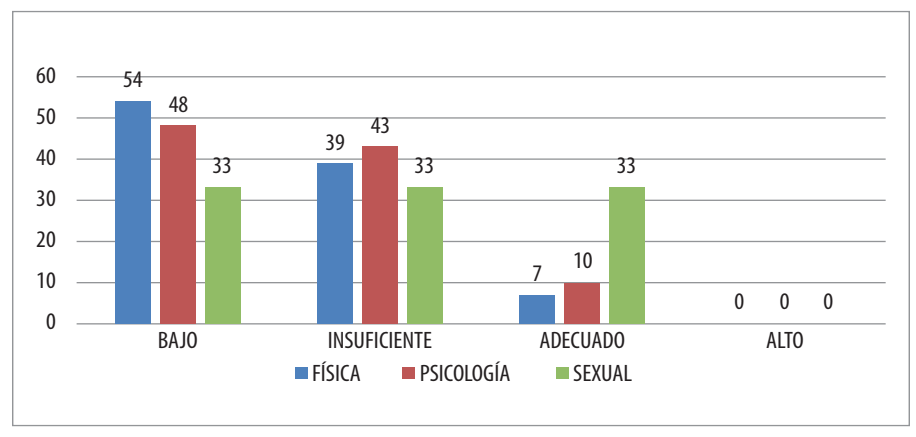

En cuanto a la violencia, de todas las mujeres encuestadas, se encontró que en algún momento sufrieron algún tipo violencia familiar. Los resultados del estudio evidenciaron que la violencia psicológica estuvo presente en todos los casos, de los cuales el $48 \%$ de ellas tuvieron un RN con bajo peso para la edad gestacional, y solo un $10 \%$ de las madres que sufrieron violencia psicológica tuvieron como producto recién nacidos adecuados para la edad gestacional,. En segundo lugar se encontró que 28 mujeres encuestadas presentaron violencia física y que el $54 \%$ de ellas tuvieron un RN con bajo peso para la edad gestacional y un $7 \%$ de ellas lograron recién nacidos con peso adecuado para la edad gestacional. Y en último lugar se encontró 3 mujeres que sufrieron violencia sexual y de ellas un recién nacido con bajo peso al nacer.

\section{RESULTADOS Y DISCUSIÓN}

Con respecto a los datos generales de las madres encuestadas, la edad promedio que se encontró fue de 27 años considerándose una población joven, lo cual coincide con la Encuesta demográfica y de salud familiar del 2013, donde nos muestran que la violencia familiar es más frecuente entre las edades de 25 a 29 años $(73.9 \%)$, este dato es importante resaltarlo puesto que si hablamos de violencia familiar, a población que está siendo afectada por este problema en la actualidad, son poblaciones cada vez más jóvenes, dato preocupante ya que nos referimos a mujeres embarazadas y que este grupo etario es la mejor edad en términos biológicos para ser madre, las condiciones físicas de la mujer dan las condiciones más favorables para concebir, engendrar y tener un parto adecuado con un hijo en buenas condiciones 
de salud. Por otro lado también se estudió a la educación, como factor de riesgo para presentar violencia, observándose que la mayoría de mujeres $(25 \%)$ no habrían concluido la secundaria. mientras que un mínimo porcentaje habría culminado estudios superiores, datos que coligen con ENDES 2013, que el 70.4\% de las mujeres violentadas han terminado el nivel secundario, resultados que nos hacen evidenciar que las mujeres con poca educación tienen un factor de riesgo para ser violentadas. En cuanto al estado civil, un 95\% tenían pareja mientras que solo un $5 \%$ eran solteras, lo cual no significa necesariamente que no presentaron violencia por no convivir con una pareja, ya que si tenían una relación con el padre de sus hijos, estos datos encontrados coinciden con los resultados de ENDES 2013 que consideran el estado conyugal de separada o divorciada no fue factor protector de violencia familiar puesto que a nivel nacional el $74 \%$ de mujeres violentadas tenían las condiciones conyugales antes mencionadas, además de otros factores que derivan de ella como la ocupación. Con estos resultados afirmamos que estos factores de riesgo intervienen en la violencia puestos que las mujeres maltratadas suelen tener inhibiciones para hablar de este problema, entre los datos encontrados y los de mayor preocupación se observa que todas las mujeres en alguna oportunidad sufrieron el tipo de violencia psicológica, seguido de la violencia física y en último lugar la violencia sexual, lo que influye en la presencia de recién nacidos con bajo peso al nacer, o con un peso inadecuado para su edad gestacional.

\section{CONCLUSIONES}

1. Las características de las madres de los recién nacidos son de alguna manera factores de riesgo para presentar violencia durante el embarazo, entre ellos edad, grado de instrucción y estado civil.

2. El peso promedio de los recién nacidos fue de $2.5125 \mathrm{~g}$, todos ellos nacidos a término, encontrándose en el límite inferior para clasificarlo como peso insuficiente.

3. Dentro de los distintos tipos de violencia estudiados, vemos que cada una de ellas guarda cierto tipo de relación con el peso del recién nacido, en donde la presencia de violencia física durante el embarazo confir- mo que el $54 \%$ de los RN obtuvieron bajo peso al nacer, , mientras que la presencia de violencia psicológica en las madres trajo como consecuencia que el $40 \%$ de los $\mathrm{RN}$ terminaron con bajo peso para la edad gestacional, así mismo la violencia sexual influye en el peso del recién nacido puesto que de los tres casos observados, 2 de ellos tienen alteración del peso de recién nacido.

4. Existe una relacion entre la violencia tanto fisica, psicologica y sexual durante el embarazo y el peso del recien nacido, en donde se afirma que existe mas probabilidad de presentar un recien nacido con bajo peso para la edad gestacional, si la madre ha sufrido algun tipo de violencia.

\section{REFERENCIAS BIBLIOGRÁFICAS}

1. Serrano NC, Páez MC, Martínez MP, Casas JP, Gil L, Navarro A. Bases genéticas y moleculares de la preeclampsia. MEDUNAB 2002; 5 (15): 185-94.

2. Plan de choque para la reducción de la mortalidad materna. Ministerio de la Protección Social 2003.

3. Saboya MI. Mortalidad materna en Colombia: Una aproximación ecológica. Universidad Nacional de Colombia. Tesis de grado para magister en salud pública. 2005.

4. Boada CL y Cotes M. Plan de choque para la reducción de la mortalidad materna. Ministerio de la Protección Social. 2004.

5. OPS 2002. Publicado en: www. paho.org.

6. DANE. Dirección de Censos y Demografía. Estadísticas vitales. Registro de nacimientos y defunciones del año 2001. Los datos de mortalidad son crudos, validados sin ajustar por subregistros.

7. DANE. Dirección de Censos y Demografía. Estadísticas vitales. Archivo de nacidos vivos y defunciones (2001), por departamento de residencia.

8. Bautista A. Hipertensión y embarazo. Toxemia gravídica. En: Ñáñez $\mathrm{H}$, Ruiz Al, eds. Texto de obstetricia y perinatologia Una contribución a la enseñanza del arte, ciencia y tecnologia. Pharmacia Upjohn. 1999. Capítulo 33, 487-524.

9. CIE-10. Clasificación Estadística Internacional de Enfermedades y Problemas relacionados. 